\title{
LAGUERRE AND MEIXNER POLYNOMIALS IN DUALITY
}

\author{
FRANCISZEK HUGON SZAFRANIEC \\ Instytut Matematyki, Uniwersytet Jagielloński, ul. Reymonta 4, PL-30059 Kraków \\ e-mail:fhszafra@im.uj.edu.pl
}

(Received 30 October, 2003; accepted 5 April, 2004)

\begin{abstract}
A closer look at Laguerre and Meixner polynomials shows that they interact, with a more active role played by the latter. We intend here to expound on this development of the story up to some level of abstraction.

2000 Mathematics Subject Classification. 33C45.

Both Laguerre and Meixner polynomials belong to the classical part of the theory of orthogonal polynomials. While Laguerre polynomials have found a firm position in theoretical physics (exemplum: the hydrogen atom), the Meixner ones have rather been put aside. It turns out that if one passes from Laguerre polynomials to Laguerre functions (they are orthonormal and complete in $\mathcal{L}^{2}\left(\mathbb{R}_{+}\right)$) on the one hand and from Meixner polynomials to Meixner sequences (which are orthonormal and complete in $\ell^{2}$ ) on the other, the two meet in perfect harmony (if not to say, coincide, at least from the point of view of the Hilbert space category); this theme is developed in [9]. Here we intend to show how they interplay. This kind of interplay was discovered in [5] as a property of Hilbert space operators and then settled in the proper environment of special functions in [6] and [7].
\end{abstract}

The differential operators. The Laguerre polynomials $L_{n}^{(\alpha)}, n=0,1, \ldots, \alpha>-1$, can be given ${ }^{1}$ as

$$
L_{n}^{(\alpha)}(x) \stackrel{\mathrm{df}}{=} \frac{(\alpha)_{n}}{n !}{ }_{1} F_{1}\left(\begin{array}{c}
-n \\
\alpha+1
\end{array} \mid x\right)
$$

They satisfy ${ }^{2}[\mathbf{1 0}]$

$$
(n+1) L_{n+1}^{(\alpha)}(x)=\left(-x D^{2}-(\alpha+1-2 x) D+\alpha+1-x\right) L_{n}^{(\alpha)}(x), n=0,1, \ldots
$$

which is the raising formula for them ${ }^{3}$. The other, the lowering formula [8] is ${ }^{4}$ (with convention $L_{-1}^{(\alpha)}=0$ )

$$
(n+\alpha) L_{n-1}^{(\alpha)}(x)=\left(-x D^{2}-(\alpha+1) D\right) L_{n}^{(\alpha)}(x), \quad n=0,1, \ldots
$$

\footnotetext{
At some stage this work was supported by the KBN grant 2 P03A 037024.

${ }^{1}(a)_{n}$ defined as $(a)_{0} \stackrel{\mathrm{df}}{=} 1$ and $(a)_{n} \stackrel{\mathrm{df}}{=}(a)_{n-1}(a+n-1)$ stands for the shifted factorial (the Pochhammer symbol).

${ }^{2}$ Occasionally, depending on the context, we use the shorthand notation $D \stackrel{\mathrm{df}}{=} \frac{\mathrm{d}}{\mathrm{d} x}$.

${ }^{3}$ We distinguish between operational formulae and Hilbert space operators; this is to avoid messy exposition.

${ }^{4}$ There is a misprint in formula (2) (as wel as in (4)) in [8]; +1 has to be dropped in both.
} 
Recalling the orthogonality relation

$$
\int_{0}^{\infty} L_{m}^{(\alpha)}(x) L_{n}^{(\alpha)}(x) x^{\alpha} e^{-x} \mathrm{~d} x=\frac{\Gamma(n+\alpha+1)}{n !} \delta_{m, n}, \quad m, n=0,1, \ldots
$$

and defining the Laguerre functions

$$
l_{n}^{(\alpha)}(x) \stackrel{\mathrm{df}}{=} \sqrt{\frac{n !}{\Gamma(n+\alpha+1)}} x^{\alpha / 2} e^{-x / 2} L_{n}^{(\alpha)}(x), \quad x \geq 0, \quad n=0,1, \ldots
$$

we get from (1) for $n=0,1, \ldots$ the following formula

$$
\sqrt{(n+1)(n+\alpha+1)} l_{n+1}^{(\alpha)}(x)=\left(-x D^{2}+(x-1) D-\frac{x^{2}-2 x-\alpha^{2}}{4 x}\right) l_{n}^{(\alpha)}(x) .
$$

Since the sequence $\left\{L_{n}^{(\alpha)}\right\}_{n=0}^{\infty}$ is complete in $\mathcal{L}^{2}\left(\mathbb{R}_{+}, x^{\alpha} e^{-x} \mathrm{~d} x\right)$, the sequence $\left\{l_{n}^{(\alpha)}\right\}_{n=0}^{\infty}$ forms an orthonormal basis in $\mathcal{L}^{2}\left(\mathbb{R}_{+}\right)$(with $\mathbb{R}_{+}$for $[0,+\infty)$ ). Thus we come from (2) to a family $S_{\alpha}, \alpha>-1$, of densely defined operators in $\mathcal{L}^{2}\left(\mathbb{R}_{+}\right)$defined as

$$
\begin{aligned}
\left(S_{\alpha} f\right)(x) & \stackrel{\mathrm{df}}{=}\left(-x \frac{\mathrm{d}^{2}}{\mathrm{~d} x^{2}}+(x-1) \frac{\mathrm{d}}{\mathrm{d} x}+\frac{-x^{2}+2 x+\alpha^{2}}{4 x}\right) f(x), \\
f \in \mathcal{D}\left(S_{\alpha}\right) & \stackrel{\mathrm{df}}{=} \operatorname{lin}\left\{l_{n}^{(\alpha)} ; n=0,1, \ldots\right\},
\end{aligned}
$$

which act as forward weighted shift operators (with respect to the basis $\left\{l_{n}^{(\alpha)}\right\}_{n=0}^{\infty}$ ), that is

$$
S_{\alpha} l_{n}^{(\alpha)}=\sqrt{(n+1)(n+\alpha+1)} l_{n+1}^{(\alpha)}, n=0,1, \ldots
$$

The Hilbert space adjoint $S_{\alpha}^{*}$ of $S_{\alpha}$, acts for $f \in \operatorname{lin}\left\{l_{n}^{(\alpha)} ; n=0,1, \ldots\right\}$ as

$$
\left(S_{\alpha}^{*} f\right)(x) \stackrel{\mathrm{df}}{=}\left(-x \frac{\mathrm{d}^{2}}{\mathrm{~d} x^{2}}-(x+1) \frac{\mathrm{d}}{\mathrm{d} x}+\frac{-x^{2}-2 x+\alpha^{2}}{4 x}\right) f(x) .
$$

It is a backward weighted shift with respect to the Laguerre functions

$$
S_{\alpha}^{*} l_{n}^{(\alpha)}= \begin{cases}\sqrt{n(n+\alpha)} l_{n+1}^{(\alpha)} & \text { if } n=1,2, \ldots \\ 0 & \text { if } n=0\end{cases}
$$

Both $S_{\alpha}$ and $S_{\alpha}^{*}$ are the ladder operators for the Laguerre polynomials.

The finite difference operators. The Meixner polynomials $M_{n}^{(\beta, c)}, n=0,1, \ldots$, with parameters $\beta>0$ and $0<c<1$, can be defined (cf. [3]) as

$$
M_{n}^{(\beta, c)}(x) \stackrel{\mathrm{df}}{=}{ }_{2} F_{1}\left(\begin{array}{c}
-n,-x \\
\beta
\end{array} \mid 1-\frac{1}{c}\right)
$$

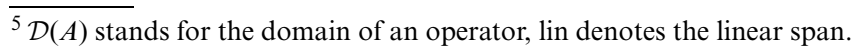


They are related to their generating function as follows

$$
\left(1-\frac{t}{c}\right)^{x}(1-t)^{-x-\beta}=\sum_{n=0}^{\infty}(\beta)_{n} M_{n}^{(\beta, c)}(x) \frac{t^{n}}{n !},
$$

Write (6) for $x+1$ instead of $x$ and put these two together so as to get

$$
\left(1-\frac{t}{c}\right) \sum_{n=0}^{\infty}(\beta)_{n} M_{n}^{(\beta, c)}(x) \frac{t^{n}}{n !}=(1-t) \sum_{n=0}^{\infty}(\beta)_{n} M_{n}^{(\beta, c)}(x+1) \frac{t^{n}}{n !} .
$$

Comparing the coefficients at $t^{n}$ we arrive at

$$
(\beta+n-1)\left[M_{n+1}^{(\beta, c)}(x)-M_{n+1}^{(\beta, c)}(x+1)\right]=n\left(\frac{1}{c} M_{n}^{(\beta, c)}(x)-M_{n}^{(\beta, c)}(x+1)\right) .
$$

On the other hand, the difference relation for the Meixner polynomials is

$$
\begin{aligned}
-n M_{n}^{(\beta, c)}(x)= & \frac{c}{1-c}(x+\beta) M_{n}^{(\beta, c)}(x+1) \\
& -\frac{x+(x+\beta) c}{1-c} M_{n}^{(\beta, c)}(x)+\frac{x}{1-c} M_{n}^{(\beta, c)}(x-1) .
\end{aligned}
$$

Changing $n$ for $n+1$ we can rewrite the above difference relation as

$$
\begin{aligned}
-(n+1) M_{n+1}^{(\beta, c)}(x)= & \frac{c}{1-c}(x+\beta)\left(M_{n+1}^{(\beta, c)}(x+1)-M_{n+1}^{(\beta, c)}(x)\right) \\
& -\frac{x}{1-c}\left(M_{n+1}^{(\beta, c)}(x)-M_{n+1}^{(\beta, c)}(x-1)\right)
\end{aligned}
$$

and inserting (7) into the brackets of (8) we get

$$
\begin{aligned}
-(n+1) M_{n+1}^{(\beta, c)}(x)= & \frac{c}{1-c}(x+\beta) \frac{n+1}{\beta+n}\left(M_{n}^{(\beta, c)}(x+1)-c^{-1} M_{n}^{(\beta, c)}(x)\right) \\
& -\frac{x}{1-c} \frac{n+1}{\beta+n}\left(M_{n}^{(\beta, c)}(x)-c^{-1} M_{n}^{(\beta, c)}(x-1)\right) .
\end{aligned}
$$

Finally, the raising formula for Meixner polynomials is

$$
\begin{aligned}
(\beta+n) M_{n+1}^{(\beta, c)}(x)= & \frac{1}{1-c}\left((2 x+\beta) M_{n}^{(\beta, c)}(x)-c(x+\beta) M_{n}^{(\beta, c)}(x+1)\right. \\
& \left.+-c^{-1} x M_{n}^{(\beta, c)}(x-1)\right), \quad n=0,1, \ldots
\end{aligned}
$$

Recall that the orthogonality relation for Meixner polynomials is of the form

$$
\sum_{x=0}^{\infty} \frac{(\beta)_{x}}{x !} c^{x} M_{k}^{(\beta, c)}(x) M_{l}^{(\beta, c)}(x)=\frac{c^{-k} k !}{(\beta)_{k}(1-c)^{\beta}} \delta_{k l}
$$

Define the $n$-th Meixner sequence as

$$
m_{n}^{(\beta, c)}(x) \stackrel{\mathrm{df}}{=}(1-c)^{\beta / 2} \sqrt{\frac{(\beta)_{n} c^{n}}{n !}} \sqrt{\frac{(\beta)_{x} c^{x}}{x !}} M_{n}^{(\beta, c)}(x), \quad x=0,1, \ldots
$$


Then (9) takes the form (notice here $m_{n}^{(\beta, c)}(-1)$ or, rather, the whole expression in the second bracket is irrelevant)

$$
\begin{aligned}
\sqrt{(n+1)(\beta+n)} m_{n+1}^{(\beta, c)}(x)= & \frac{1}{1-c}\left((2 x+\beta) \sqrt{c} m_{n}^{(\beta, c)}(x)-c \sqrt{(x+1)(x+\beta)} m_{n}^{(\beta, c)}(x+1)\right. \\
& \left.-\sqrt{x(x+\beta-1)} m_{n}^{(\beta, c)}(x-1)\right) .
\end{aligned}
$$

Because, due to (10) each $m_{n}^{(\beta, c)}$ is in $\ell^{2}$, we can define an operator $S_{\beta, c}$ in $\ell^{2}$ as follows: $\mathcal{D}\left(S_{\beta, c}\right) \stackrel{\mathrm{df}}{=} \operatorname{lin}\left\{m_{n}^{(\beta, c)} ; n=0,1, \ldots\right\}$ and for $f \in \mathcal{D}\left(S_{\beta, c}\right)$,

$$
\begin{aligned}
\left(S_{\beta, c} f\right)(x) \stackrel{\mathrm{df}}{=} & \frac{1}{1-c}(\sqrt{c}(2 x+\beta) f(x)-c \sqrt{(x+1)(x+\beta)} f(x+1) \\
& -\sqrt{x(x+\beta-1)} f(x-1)), \quad x=1,2, \ldots, \\
\left(S_{\beta, c} f\right)(0) \stackrel{\mathrm{df}}{=} & \frac{1}{1-c}(c \beta f(0)-c \sqrt{\beta} f(1)) .
\end{aligned}
$$

A look at (11) and (5) convinces us that

$$
m_{n}^{(\beta, c)}(x)=m_{x}^{(\beta, c)}(n), \quad n, x=0,1, \ldots
$$

Because of this selfduality, by [2], the sequence $\left\{m_{n}^{(\beta, c)}\right\}_{n=0}^{\infty}$ forms a basis, that is an orthonormal and complete set, in $\ell^{2}$. Thus $S_{\beta, c}$ is a densely defined operator in $\ell^{2}$ and

$$
S_{\beta, c} m_{n}^{(\beta, c)}=\sqrt{(n+1)(n+\beta)} m_{n+1}^{(\beta, c)}, \quad n=0,1, \ldots
$$

Consequently, its adjoint $S_{\beta, c}^{*}$ acts on $\operatorname{lin}\left\{m_{n}^{(\beta, c)} ; n=0,1, \ldots\right\}$ as (cf. [4])

$$
S_{\beta, c}^{*} m_{n}^{(\beta, c)}= \begin{cases}\sqrt{n(n+\beta-1)} m_{n-1}^{(\beta, c)} & \text { if } n=1,2, \ldots \\ 0 & \text { if } n=0\end{cases}
$$

and its finite difference form is

$$
\begin{aligned}
\left(S_{\beta, c}^{*} f\right)(x)= & \frac{1}{1-c}(\sqrt{c}(2 x+\beta) f(x)-c \sqrt{x(x+\beta-1)} f(x-1) \\
& -\sqrt{(x+1)(x+\beta)} f(x+1)), \quad x=1,2, \ldots, \\
\left(S_{\beta, c}^{*} f\right)(0)= & \frac{1}{1-c}(\sqrt{c} \beta f(0)-\sqrt{\beta} f(1)) .
\end{aligned}
$$

Notice that this formula extends to the whole of $\mathcal{D}\left(S_{\beta, c}^{*}\right)$ and that $\mathcal{D}\left(\bar{S}_{\beta, c}\right)=\mathcal{D}\left(S_{\beta, c}^{*}\right)$, where $\bar{S}_{\beta, c}$ is the closure of $S_{\beta, c}$.

Invoking the defining formula (11) we get from the above and (14)

$$
\begin{aligned}
n M_{n-1}^{(\beta, c)}(x)= & \frac{c}{1-c}\left((2 x+\beta) M_{n}^{(\beta, c)}(x)-(x+\beta) M_{n}^{(\beta, c)}(x+1)\right. \\
& \left.-x M_{n}^{(\beta, c)}(x-1)\right), \quad n=1,2, \ldots
\end{aligned}
$$

and this is the lowering formula ${ }^{6}$ for Meixner polynomials.

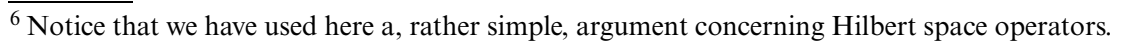


Let $\left\{\epsilon_{n}\right\}_{n=0}^{\infty}$ be the canonical zero-one basis in $\ell^{2}$, that is $\epsilon_{n}=\left\{\delta_{n, i}\right\}_{i=0}^{\infty}$. Because $\left\{\epsilon_{n}\right\}_{n=0}^{\infty} \subset$ $\mathcal{D}\left(\bar{S}_{\beta, c}\right)$ we have

$$
\begin{aligned}
\bar{S}_{\beta, c} \epsilon_{n}= & \frac{1}{1-c}\left(\sqrt{c}(2 n+\beta) \epsilon_{n}-c \sqrt{n(n+\beta-1)} \epsilon_{n-1}\right. \\
& \left.-\sqrt{(n+1)(n+\beta)} \epsilon_{n+1}\right), \quad n=1,2, \ldots, \\
\bar{S}_{\beta, c} \epsilon_{0}= & \frac{1}{1-c}\left(c \beta \epsilon_{0}-\sqrt{\beta} \epsilon_{1}\right) .
\end{aligned}
$$

and

$$
\begin{aligned}
S_{\beta, c}^{*} \epsilon_{n}= & \frac{1}{1-c}\left(\sqrt{c}(2 n+\beta) \epsilon_{n}-c \sqrt{(n+1)(n+\beta)} \epsilon_{n+1}\right. \\
& \left.-\sqrt{n(n+\beta-1)} \epsilon_{n-1}\right), \quad x=1,2, \ldots, \\
S_{\beta, c}^{*} \epsilon_{0}= & \frac{1}{1-c}\left(\sqrt{c} \beta \epsilon_{0}-\sqrt{\beta} \epsilon_{1}\right) .
\end{aligned}
$$

The abstract setup. Let $\mathcal{H}$ be a separable Hilbert space with the inner product $\langle\cdot,-\rangle$ and let $\left\{e_{n}\right\}_{n=0}^{\infty}$ be a basis in it. Set

$$
e_{n}^{(\beta, c)} \stackrel{\mathrm{df}}{=} \sum_{k=0}^{\infty} m_{k}^{(\beta, c)}(n) e_{k} ; n=0,1, \ldots
$$

Then, by (13) and according to Proposition 2 of [6], $\boldsymbol{e}^{(\beta, c)} \stackrel{\mathrm{df}}{=}\left\{e_{n}^{(\beta, c)}\right\}_{n=0}^{\infty}$ is another basis in $\mathcal{H}$ and the reciprocity

$$
e_{n}=\sum_{k=0}^{\infty} m_{n}^{(\beta, c)}(k) e_{k}^{(\beta, c)} ; n=0,1, \ldots
$$

holds.

A densely defined closable operator $S$ in $\mathcal{H}$ is said to be a (forward) weighted shift with respect to $e$ and with the weights $\left\{\sigma_{n}\right\}_{n=0}^{\infty}$ if $\operatorname{lin}\left\{e_{n} ; n=0,1, \ldots\right\}$ is a core ${ }^{7}$ of $S$, $S e_{n}=\sigma_{n} e_{n+1}$ for $n=0,1, \ldots$ and all $\sigma_{n}$ 's are positive numbers. In like manner, an operator $T$ is called a backward weighted shift with respect to $\boldsymbol{e}$ and with the weights $\left\{\tau_{n}\right\}_{n=0}^{\infty}$ if $\operatorname{lin}\left\{e_{n} ; n=0,1, \ldots\right\}$ is a core of $T, T e_{n}=\tau_{n-1} e_{n-1}$ for $n=0,1, \ldots$ and all $\tau_{n}$ 's are positive numbers. If all $\sigma_{n}=\tau_{n}$ the operators $S$ and $T$ are formally adjoint each to the other, that is $\langle S f, g\rangle=\langle f, T g\rangle$, for $f, g \in \operatorname{lin}\left\{e_{n} ; n=0,1, \ldots\right\}$; in fact we always have $\mathcal{D}(S) \subset \mathcal{D}\left(S^{*}\right)$ and the inclusion may be strict in general, cf. [4].

Prompted by the presentation so far we are going to distinguish two weighted shifts, both with the same weights $\sigma_{n}=\sqrt{(n+1)(n+\beta)}$ with $\beta>0$ : the forward weighted shift with these weights $\boldsymbol{S}_{\beta, c}$ and the related backward shift which is nothing, as we already know, but the adjoint $\boldsymbol{S}_{\beta, c}^{*}$ restricted to $\operatorname{lin}\left\{e_{n} ; n=0,1, \ldots\right\}$. They are a kind of creation and annihilation operators in our circumstances and we would like to hold on these names here. According to what we have said it is enough to consider one of them, the creation operator say. Fortunately in this case $\mathcal{D}\left(\overline{\boldsymbol{S}}_{\beta, c}\right)=\mathcal{D}\left(\boldsymbol{S}_{\beta, c}^{*}\right)$.

$\overline{{ }^{7} \mathcal{D} \subset \mathcal{D}(A)}$ is a core of a closable operator $A$ if $\overline{\left.A\right|_{\mathcal{D}}}=\bar{A}$ with ${ }^{-}$standing for the closure. 
Set

$$
M \stackrel{\mathrm{df}}{=} \operatorname{diag}(n)_{n=0}^{\infty} \text { with respect to the basis } \boldsymbol{e} .
$$

Using closability of $M$, by (15), we infer that $\boldsymbol{e}^{(\beta, c)} \subset \mathcal{D}(\bar{M})$. Also $f_{n} \stackrel{\mathrm{df}}{=} \sqrt{(N+1)(N+\beta)}$ $\times\left[m_{N}^{(\beta, c)}(n) e_{N+1}-c m_{N+1}^{(\beta, c)}(n) e_{N}\right]$. By (12) we have ${ }^{8}\left(\right.$ with $e_{-1}=0$ and $m_{k}^{(\beta, c)}(-1) \stackrel{\text { df }}{=} 0$ )

$$
\begin{gathered}
{\left[\boldsymbol{S}_{\beta, c}+c \boldsymbol{S}_{\beta, c}^{*}-\beta \sqrt{c} I-2 \sqrt{c} M\right] \sum_{k=0}^{N} m_{k}^{(\beta, c)}(n) e_{k}} \\
\stackrel{(12)}{=} \sum_{k=0}^{N} m_{k}^{(\beta, c)}(n)\left[\sqrt{(k+1)(k+\beta)} e_{k+1}+c \sqrt{k(k+\beta-1)} e_{k-1}-\sqrt{c}(2 k+\beta) e_{k}\right] \\
=\sum_{i=0}^{N}\left[\sqrt{i(i+\beta-1)} m_{n}^{(\beta, c)}(i-1)+c \sqrt{(i+1)(i+\beta)} m_{n}^{(\beta, c)}(i+1)\right. \\
\left.-\sqrt{c}(2 i+\beta) m_{n}^{(\beta, c)}(i)\right] e_{i}+f_{N} \\
\stackrel{(12)}{=}-(1-c) \sqrt{(n+1)(n+\beta)} \sum_{i=0}^{N} m_{n+1}^{(\beta, c)}(i) e_{i}+f_{N} .
\end{gathered}
$$

Because $f_{N} \rightarrow 0, \quad \sum_{k=0}^{N} m_{k}^{(\beta, c)}(n) e_{k} \rightarrow e_{n}^{(\beta, c)}$ and $\boldsymbol{S}_{\beta, c} \sum_{k=0}^{N} m_{k}^{(\beta, c)}(n) e_{k} \rightarrow \sqrt{c}(2 n+$ $\beta) e_{n}^{(\beta, c)}-c \sqrt{n(n+\beta-1)} e_{n-1}^{(\beta, c)}-\sqrt{(n+1)(n+\beta)} e_{n+1}^{(\beta, c)}$ as $N \rightarrow \infty$ we get therefrom $e_{n}^{(\beta, c)} \in \mathcal{D}\left(\overline{\boldsymbol{S}}_{\beta, c}\right)$ and

$$
\left(\overline{\boldsymbol{S}}_{\beta, c}+c \boldsymbol{S}_{\beta, c}^{*}-\beta \sqrt{c} I-2 \sqrt{c} \bar{M}\right) e_{n}^{(\beta, c)}=-(1-c) \sqrt{(n+1)(n+\beta)} e_{n+1}^{(\beta, c)} .
$$

Let $U_{\beta, c}$ be a unitary operator sending $e_{n}$ 's to $e_{n}^{(\beta, c)}$ 's. Then (1) can be read as

$$
\boldsymbol{S}_{\beta, c}=-\frac{1}{1-c} U_{\beta, c}^{*}\left(\overline{\boldsymbol{S}}_{\beta, c}+c \boldsymbol{S}_{\beta, c}^{*}-\beta \sqrt{c} I-2 \sqrt{c} \bar{M}\right) U_{\beta, c}
$$

Let us record this as

THEOREM 1. The creation operator $\boldsymbol{S}_{\beta, c}$ satisfies (1) or, equivalently, (19).

The one before last and the last component of (17) can be now stated as

THEOREM 2. The creation operators $\boldsymbol{S}_{\beta, c}$ in $\mathcal{H}$ and $S_{\beta, c}$ in $\ell^{2}$ satisfy

$$
\boldsymbol{S}_{\beta, c} e_{n}^{(\beta, c)}=-\frac{1}{1-c} \sum_{k=0}^{\infty}\left(S_{\beta, c} m_{n}^{(\beta, c)}\right)(k) e_{k}, \quad n=0,1, \ldots
$$

As a next step we work out a kind of converse to these Theorems.

The punchline. Now we take delight in convincing ourselves that the operators $\boldsymbol{S}_{\beta, c}$ and $\boldsymbol{S}_{\beta, c}$, appearing in Theorems 1 and 2, are the only possible solutions of (19) and (20) within the class of weighted shifts.

$\overline{{ }^{8} \text { Here and }}$ in the sequel we make constant use of (13) without mentioning it. 
Fix $c$ and $\beta$ and suppose we are given

$1^{\circ}$ two bases $\boldsymbol{e}=\left\{e_{n}\right\}_{n=0}^{\infty}$ and $\boldsymbol{f}=\left\{f_{n}\right\}_{n=0}^{\infty}$

$2^{\circ}$ two closable operators $S$ and $\boldsymbol{T}$ such that line is a core for $\boldsymbol{S}$ and so is lin $\boldsymbol{e}^{(\beta, c)}$ for $\boldsymbol{T}$, and such that $\boldsymbol{e}^{(\beta, c)} \subset \mathcal{D}(\boldsymbol{S}) \cap \mathcal{D}\left(\boldsymbol{S}^{*}\right)$ and $\boldsymbol{e}, \boldsymbol{f} \subset \mathcal{D}(\boldsymbol{T})$.

By analogy with (19) suppose (with $M$ defined by (3) and the remark afterwards)

$$
\boldsymbol{T} e_{n}=-\frac{1}{1-c}\left(\boldsymbol{S}+c \boldsymbol{S}^{*}-\beta \sqrt{c} I-2 \sqrt{c} \bar{M}\right) e_{n}, \quad n=0,1, \ldots
$$

THEOREM 3. Suppose for some $\beta>0$ and $0<c<1 \boldsymbol{S}$ is a weighted shift with respect to $\boldsymbol{e}$ with some weights $\left\{\sigma_{n}\right\}_{n=0}^{\infty}$ and so is $\boldsymbol{T}$ with respect to $\boldsymbol{f}$ with some other weights $\left\{\tau_{n}\right\}_{n=0}^{\infty}$. Then $\boldsymbol{f}=\boldsymbol{e}^{(\beta, c)}$ and $\boldsymbol{S}$ is a creation operator with respect to $\boldsymbol{e}$ and at the same time so is $\boldsymbol{T}$ with respect to $\boldsymbol{e}^{(\beta, c)}$, all this provided $\sigma_{0}=\tau_{0}=1$ and $f_{0}=e_{0}^{(\beta, c)}$.

Proof. Making use of closability of the operators involved and the very specific way they act, as we have done it so far, we get (suppose the weights for $\boldsymbol{S}$ and $\boldsymbol{T}$ are $\sigma_{n}$ 's and $\tau_{n}$ 's resp.)

$$
\begin{aligned}
& \text { LHS of (4) }=\sum_{i=0}^{\infty}\left\langle e_{n}, f_{i}\right\rangle \tau_{i} f_{i}=\sum_{i, j=0}^{\infty}\left\langle e_{n}, f_{i}\right\rangle \tau_{i}\left\langle f_{i}, e_{j}\right\rangle e_{j}, \\
& \text { RHS of }(3)=-(1-c)^{-1}\left(\sigma_{n} e_{n+1}+\sigma_{n-1} e_{n-1}-\sqrt{c}(2 n+\beta) e_{n}\right) .
\end{aligned}
$$

Taking into account the fact that, due to (13), $\left\langle e_{k}^{(\beta, c)}, e_{l}\right\rangle=m_{k}^{(\beta, c)}(l)=m_{l}^{(\beta, c)}(k)$ we get from these two for the inner product with $e_{k}^{(\beta, c)}$

$$
\begin{aligned}
& \sum_{i, j=0}^{\infty}\left\langle e_{n}, f_{i}\right\rangle \tau_{i}\left\langle f_{i}, e_{j}\right\rangle m_{k}^{(\beta, c)}(j) \\
& \quad=-(1-c)^{-1}\left(\sigma_{n} m_{k}^{(\beta, c)}(n+1)+\sigma_{n-1} m_{k}^{(\beta, c)}(n-1)-\sqrt{c}(2 n+\beta) m_{k}^{(\beta, c)}(n)\right) .
\end{aligned}
$$

Comparing this with (12) and starting with the initial conditions $\sigma_{0}=\tau_{0}=1$ and $f_{0}=e_{0}^{(\beta, c)}$ we come to the final conclusion.

The next result refers to (20) we state one of the possibilities, the proof as well as other versions can be pattern after [7]. Consider the following two conditions

$$
\begin{aligned}
S e_{n}^{(\beta, c)} & =-\frac{1}{1-c} \sum_{k=0}^{\infty}\left(S m_{n}^{(\beta, c)}\right)(k) e_{k}, \quad n=0,1, \ldots \\
S e_{n} & =-\frac{1}{1-c} \sum_{k=0}^{\infty}\left(S m_{n}^{(\beta, c)}\right)(k) e_{k}^{(\beta, c)}, \quad n=0,1, \ldots
\end{aligned}
$$

for $S$ to be a closable operator in $\ell^{2}$ for which $\operatorname{lin}\left\{m_{n}^{(\beta, c)} ; n=0,1, \ldots\right\}$ is a core and $\boldsymbol{S}$ an operator in $\mathcal{H}$ for which either lin $\boldsymbol{e}$ or $\operatorname{lin} \boldsymbol{e}^{(\beta, c)}$ is a core, depending on which of (22) and (23) is chosen.

THEOREM 4. Suppose $S$ is a weighted shift with respect $\left\{m_{n}^{(\beta, c)}\right\}_{n=0}^{\infty}$ with weights $\left\{\sigma_{n}\right\}_{n=0}^{\infty}$ and $\boldsymbol{S}$ is a weighted shift with respect to either $\boldsymbol{e}$ or $\boldsymbol{e}^{(\beta, c)}$ depending on choice of between (22) and (23). Then $S=S_{\beta, c}$ and $\boldsymbol{S}=\boldsymbol{S}_{\beta, c}$, that is they are respective creation operators, provided $\sigma_{0}=\tau_{0}=1$. 
"Meixner" plays with "Laguerre". For the reason touched upon at the very beginning of this paper the Laguerre polynomials are specially adapted to Meixner ones. They provide us with a specific example of a Hilbert space $\mathcal{H}$ in which the creation operator $S_{\beta, c}$ manifests itself in a natural way: according to (4), $S_{\alpha+1}$ defined by (3) acts on the Laguerre functions $l_{n}^{\alpha+1}$ as a creation operator in our abstract sense. Thus one can write down mutatis mutandis our Theorems corresponding to this concrete situation. In particular the new basis (or rather a three parameter family of) is explicitly written as

$$
\sum_{k=0}^{\infty} m_{k}^{(\beta, c)}(n) l_{k}^{\alpha+1}, \quad n=0,1, \ldots
$$

It might be interesting to notice that (20), after passing to source polynomials, which can be done with ease, according to Theorem 4, determines the couple "Laguerre Meixner" uniquely.

\section{REFERENCES}

1. T. S. Chihara, An introduction to orthogonal polynomials (Gordon and Breach, New York, 1978).

2. G. K. Eagleson, A duality relation for discrete orthogonal systems, Studia Sc. Math. Hung. 3(1968), 127-136.

3. R. Koekoek and R. F. Swarttouw, The Askey-scheme of hypergeometric orthogonal polynomials and its q-analogue, Delft University of Technology, Report of the Department of Technical Mathematics and Informatics No. 98-17 (1998).

4. J. Stochel and F. H. Szafraniec, A few assorted questions about unbounded subnormal operators, Univ. Iagel. Acta Math. 28(1991), 163-170.

5. J. Stochel and F. H. Szafraniec, A peculiarity of the creation operator, Glasgow Math. J. 44 (2001), 137-147.

6. F. H. Szafraniec, Charlier polynomials and translational invariance in the quantum harmonic oscillator, Math. Nachtr. 241 (2002), 163-169.

7. F. H. Szafraniec, Duality in the quantum harmonic oscillator, J. Phys. A: Math. Gen. 34 (2001), 10487-10492. 699-701.

8. F. H. Szafraniec, The dual of a formula of Viskov, Bull. Korean Math. Soc. 40(2003),

9. F. H. Szafraniec, Laguerre and Meixner polynomials in one: the oscillator, work in progress.

10. O. V. Viskov, L. B. Redei's identity for Laguerre polynomials [in Russian], Acta Sci. Math. (Szeged) 39 (1977), 27-28. 